\title{
Solid phase extraction of melamine from water using magnetic grapheme oxide as adsorbent
}

\author{
Ming Zhou* and Junyao Huang \\ Tianhong Measurement Institute, Zhuzhou 412036, China \\ mingquanzhou@126.com
}

\begin{abstract}
Keywords: Melamine; Magnetic graphene oxide; solid phase extraction.
Abstract Melamine can be introduced into environment via various industrial effluents, thus it is necessary to develop suitable analytical methods for determination of melamine in environmental samples. In this work, magnetic graphene oxide (MGO) was synthesized and used as the adsorbent for solid phase extraction of melamine. The parameters that affect the extraction efficiency of melamine were investigated. The optimum conditions, including sample $\mathrm{pH}$, extraction and desorption time, and eluent volume were obtained. The analytical performance of the method was investigated, and it was found that the limit of detection (LOD) for melamine was $0.15 \mu \mathrm{g} / \mathrm{L}$ and the RSD was $3.6 \%$. The proposed method was utilized in the determination of melamine in environmental water samples with recoveries were higher than $92 \%$.
\end{abstract}

\section{Introduction}

Melamine (1,3,5-triazine-2,4,6-triamine, $\left.\mathrm{C}_{3} \mathrm{H}_{6} \mathrm{~N}_{6}\right)$ is an organic compound produced in large amounts. Melamine-contaminated infant formulas have resulted in serious health problems [1-3]. Nowadays, melamine has also been present in the environment as a result of its widespread uses [4-6]. Consequently, it is necessary to develop suitable analytical methods for determination of melamine in environmental samples. Because melamine in environmental water samples is often at low levels, suitable sample preparation procedures are often needed before its determination. Among various sample preparation techniques for trace analytes, solid phase extraction is the most often used one [7-17].

Graphene oxide (GO) is the oxidized derivative of graphene, which has an ultrahigh-specific surface area, superior chemical property and physical stability. Thus, GO can be used as adsorbent materials for SPE [18-20].

Magnetically separation provides a rapid and easy route for removal of magnetic particles from solution by using a magnet [21-23]. Thus, magnetic adsorbent can be easily isolated from matrix solutions after adsorption of target analytes.

In this work, magnetic (MGO) was prepared, and applied for solid phase extraction of melamine from aqueous solution. The parameters that affect the extraction efficiency of melamine were investigated, including sample $\mathrm{pH}$, extraction time and desorption time, and eluent volume. The analytical performance of the method was examined. Finally, the developed method was applied for the determination of melamine in environmental water samples.

\section{Materials and methods}

\section{Reagents}

Melamine (>99.9\%) was obtained from Aladdin Reagent (Shanghai) Co., Ltd (Shanghai, China).Nature graphite powder (99.95\%) was purchased from Sinopharm Chemical Reagent Co., Ltd (Shanghai, China). Other chemicals and materials were purchased from Tianjin Damao Chemical Reagent Factory (Tianjin, China).

\section{Instruments}

A HPLC system (SHIMADZU, Kyoto, Japan) equipped with diode array detection (DAD, SPD-M20A) and a Shim-pack VP-ODS column $(150 \times 4.6 \mathrm{~mm}$ I.D. $)$ was utilized for analysis. 


\section{Preparation of MGO}

Graphite oxide (GO) was prepared from nature graphite powders by a modified Hummers method, and magnetic graphite oxide (MGO) was synthesized by a one-pot solvothermal reaction [16].

\section{Solid phase extraction}

$50 \mathrm{mg}$ of MGO was added to the $200 \mathrm{~mL}$ melamine containing water sample, and the solution $\mathrm{pH}$ was adjusted to $\mathrm{pH} 6$. After shaking for $20 \mathrm{~min}$, the MGO was separated from solution using a magnet. The adsorbed melamine was eluted with $3 \mathrm{~mL}$ of $6 \%$ ammonia-methanol solution.

\section{Results and discussion}

\section{Selection of solution pH}

The influence of $\mathrm{pH}$ of aqueous solution on the extraction efficiency of melamine was explored by varying $\mathrm{pH}$ value from 3 to 10. As shown in Fig.1, the recovery increased with the increase of $\mathrm{pH}$ from 3 to 5 , and then dropped slightly up to 8 . When the $\mathrm{pH}$ value was larger than 9 , the recovery decreased significantly. Therefore, $\mathrm{pH} 6$ was selected.

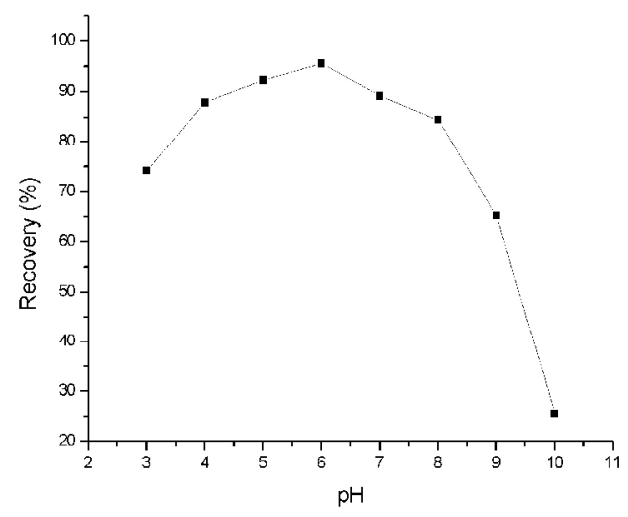

Fig.1 Effect of solution $\mathrm{pH}$.

\section{Selection of extraction time}

The effect of extraction time on extraction efficiency of melamine was investigated. As can be seen from Fig. 2, the recovery increased drastically at the initial adsorption stage from 1 to $10 \mathrm{~min}$. After 20 min, the recovery showed very little changes since the sorption equilibrium was achieved. Based on these results, 20 min was employed as the optimal extraction time.

\section{Selection of eluent volume}

In this study, 6\% ammonia-methanol solution was used as the eluent for stripping of melamine from the MGO. To examine the effect of eluent volume on the extraction recovery of melamine, different volumes of the eluent $(1,2,3,5,8$ and $10 \mathrm{~mL})$ were tested. The results showed that $3 \mathrm{~mL}$ of this solution could efficiently elute the adsorbed melamine. 


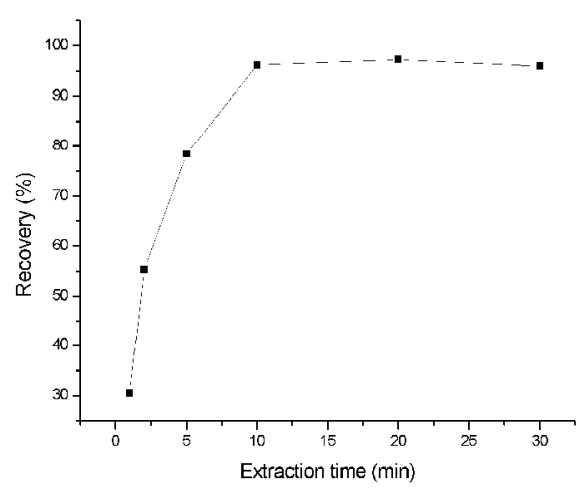

Fig.2 Effect of extraction time.

\section{Selection of desorption time}

In order to investigate the optimum desorption time, various time periods were examined in the range of 1 to $20 \mathrm{~min}$. The results were shown in Fig.3. According to Fig.3, 5 min was found to be sufficient for quantitative elution of melamine from MGO.

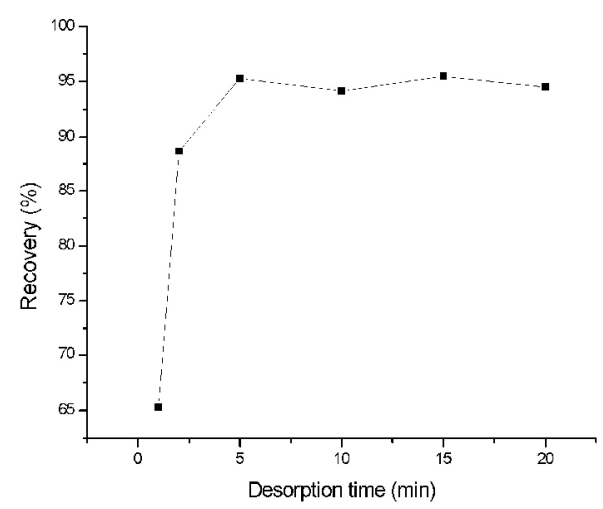

Fig.3 Effect of desorption time.

\section{Analytical performance}

Under the optimum experimental conditions, the calibration curve of melamine was linear in the concentration range from 0.3 to $30\left(R^{2}=0.9956\right)$. The limit of detection (LOD) were $0.15 \mu \mathrm{g} / \mathrm{L}$ The relative standard deviation (RSD) for 5 replicate measurements of $2.0 \mu \mathrm{g} / \mathrm{L}$ melamine was $3.6 \%$.

\section{Water analysis}

To evaluate the capability of the proposed method for analysis of real samples with different matrices containing various amounts of melamine, three environmental water samples were analyzed, and the analytical results were given in Table 1. 
Table 1 Analytical results of melamine in water samples.

\begin{tabular}{lcccc}
\hline Sample & Add $/(\mu \mathrm{g} / \mathrm{L})$ & Found $/(\mu \mathrm{g} / \mathrm{L})$ & Recovery $(\%)$ & RSD $(\%)$ \\
\hline Water sample 1 & 0 & --- & --- & 4.2 \\
& 1.0 & 0.92 & 92.0 & 3.3 \\
Water sample 2 & 0 & 8.65 & --- & 3.5 \\
Water sample 3 & 10.0 & 17.98 & 93.3 & 2.1 \\
& 0 & 65.42 & --- & 1.2 \\
& 50.0 & 113.3 & 95.8 & 2.6 \\
\hline
\end{tabular}

\section{Conclusions}

This study proposed a new method for detection of melamine in aqueous solution, using MGO based solid phase extraction coupled with HPLC-DAD. The adsorption results obtained from this study suggest that the prepared MGO can act as a suitable adsorbent for melamine. Recoveries of melamine were in the range of $92-95.8 \%$ with RSDs of $1.2-4.2 \%$, indicating the suitability of the developed method.

\section{References}

[1] Fashi A., Yaftian M.R. and Zamani A. Food Chem. 2015, 188: 92-98.

[2] Nong Y., Ma X., Fan S. and Yu Y. Anal Methods. 2014, 6(12): 4124-4129.

[3] Li R., Yang J., Han J., et al. Physica E: Low-dimensional Systems and Nanostructures, 2017, 88: 164-168.

[4] Wackett L. P., Sadowsky M. J., Martinez B., et al. Appl. Microbiol. Biotechnol. 2002, 58, 39-45.

[5] Qin Y., Lv X., Li J., et al. Environ. Int., 2010, 36, 446-452.

[6] Zhao Z., Chen L., Bai B., et al. Environ. Sci. Pollut. R. 2018, 25(3): 2285-2292.

[7] Chen J., Ma X. and Wu Y. Food Anal. Methods. 2014; 7(5): 1083-1089.

[8] Li C., Ma X., Zhang X., et al. J. Sep. Sci., 2017, 40(7): 1621-1628.

[9] Barbosa AF, Segatelli MG, Pereira AC, et al. Talanta. 2007, 71:1512-1519.

[10] Zou J., Ma X., Dang Y. and Chen Y. J Anal At Spectrom. 2014, 29(9): 1692-1697.

[11] Zhang Y., Ma X. and Fan Y. Food Anal. Methods. 2014, 7(9): 1763-1769.

[12] Ma X., Huang P. and Chen M. Rare Metals. 2007, 26(6):541-546.

[13] Li C., Ma X., Zhang X., et al.Anal. Bioanal. Chem., 2016, 408(27): 7857-7864.

[14] Heidari N.,Ghiasvand A. and Abdolhossaini S. Anal. Chim. Acta. 2017, 975:11-19.

[15] Huang R., Ma X., Li X., et al. J. Colloid Interf. Sci. 2018, 514 :544-553.

[16] Krawczyk M., Akbari S., Jeszka-Skowron M, et al. J. Anal. At. Spectrom. 2016, 31(7): 1505-1514.

[17] Fan Y., Ma X., Li Z., et al. Food Anal. Methods. 2016, 9(1): 16-22.

[18] Li D., Ma X., Wang R., et al. Anal. Bioanal. Chem. 2017, 409(5): 1165-1172.

[19] Faghihi A., Vakili M.H., Hosseinzadeh G., et al. Desalin. Water Treat. 2016, 57(47): 22655-22670.

[20] Li M., Liu Y., Liu S., et al. J. Colloid Interf. Sci. 2018, 521:150-159.

[21] Chen Y., Ma X., Huang M., Peng, J., Li C. Anal Methods. 2016; 8(4): 824-829.

[22] Hua X., You H., Luo P., et al. Anal. Bioanal. Chem. 2017, 409(29): 6885-6892.

[23] Kazemi E., Shabani A. M. H., Dadfarnia S., et al. Anal. Chim. Acta. 2016, 905: 85-92. 